\title{
Concurrent Primary Sclerosing Cholangitis and Eosinophilic Colitis
}

\author{
Naohiko Gunji, Kyoko Katakura, Atsushi Takahashi, Tatsuo Fujiwara, \\ Ryoma Suzuki, Hiroshi Watanabe and Hiromasa Ohira
}

\begin{abstract}
A 39-year-old man presented with diarrhea and abdominal pain. At 26 years of age, he was found to have eosinophilia and abnormal liver function parameters, for which prednisolone therapy was started. He subsequently underwent a liver biopsy and endoscopic retrograde cholangiopancreatography, and received a diagnosis of primary sclerosing cholangitis (PSC). On presentation to our hospital, he was further diagnosed with eosinophilic colitis based on aggravation of diarrhea and severe eosinophilic infiltration in the colonic mucosa. We herein report a rare case of concurrent PSC and eosinophilic colitis.
\end{abstract}

Key words: primary sclerosing cholangitis, eosinophilic colitis

(Intern Med 53: 2319-2324, 2014)

(DOI: 10.2169/internalmedicine.53.2765)

\section{Introduction}

Primary sclerosing cholangitis (PSC) is a relatively uncommon disease in Japan. This condition is characterized by chronic cholestasis of unknown etiology (1), and is accompanied by eosinophilia and inflammatory bowel disease, at a frequency of $39 \%$ and $37 \%$, among Japanese patients, respectively (2). In comparison, eosinophilic gastroenteritis (EG) is characterized by eosinophilic infiltration of the gastrointestinal wall with peripheral eosinophilia and produces lesions most commonly in the stomach, duodenum and small intestine and less commonly in the esophagus and large intestine (3-5). EG is known to occur concurrently with PSC and eosinophilic cholangitis, although only a few such cases have been reported. We herein report the case of a patient initially found to have abnormal liver function parameters with eosinophilia that was histologically diagnosed as PSC who subsequently developed eosinophilic colitis during the course of treatment.

\section{Case Report}

A 39-year-old man with no history of smoking or alcohol consumption presented at our hospital with abdominal pain and diarrhea. His family history included pancreatic cancer in his father and Klippel-Feil syndrome in his brother. $\mathrm{He}$ had no other contributory medical or allergic history.

In 1997 (at 26 years of age), he experienced an unusual sensation in his pharynx and consulted a physician at a nearby clinic, where he was found to have eosinophilia

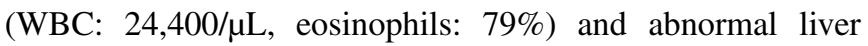
function parameters and subsequently underwent more specific examinations. His laboratory data included an aspartate aminotransferase (AST) level of $380 \mathrm{IU} / \mathrm{L}$, alanine aminotransferase (ALT) level of $270 \mathrm{IU} / \mathrm{L}$, alkaline phosphatase (ALP) level of 1,099 IU/L, gamma-glutamyltranspeptidase (GGT) level of $130 \mathrm{IU} / \mathrm{L}$, antinuclear antibody (ANA) titer of 1:2,560 (Ho), anti-smooth muscle antibody (ASMA) titer of 1: 160 and $\operatorname{IgG}$ level of $2,772 \mathrm{mg} / \mathrm{dL}$, with negative findings for anti-liver kidney microsome (aLKM)-1 antibodies, anti-mitochondrial antibodies (AMA) and hepatitis virus markers. Based on these results, a diagnosis of autoimmune hepatitis (AIH) was suspected. The human leukocyte antigen (HLA)-DR was determined to be DR4 and DR9. Although an adequate sample from the portal region was available, a liver biopsy showed only eosinophilic infiltration in the sinusoid and lobular necrotic areas, without typical findings of AIH (Fig. 1a). With respect to the eosinophilia, the IgE level was normal at $71 \mathrm{U} / \mathrm{mL}$ and 


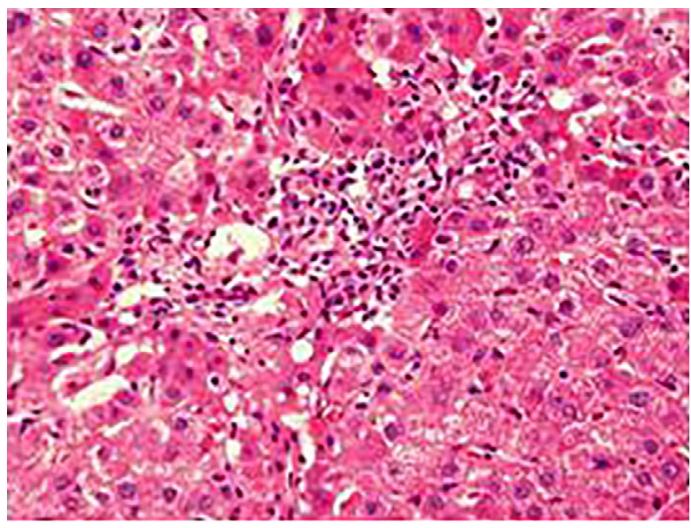

a

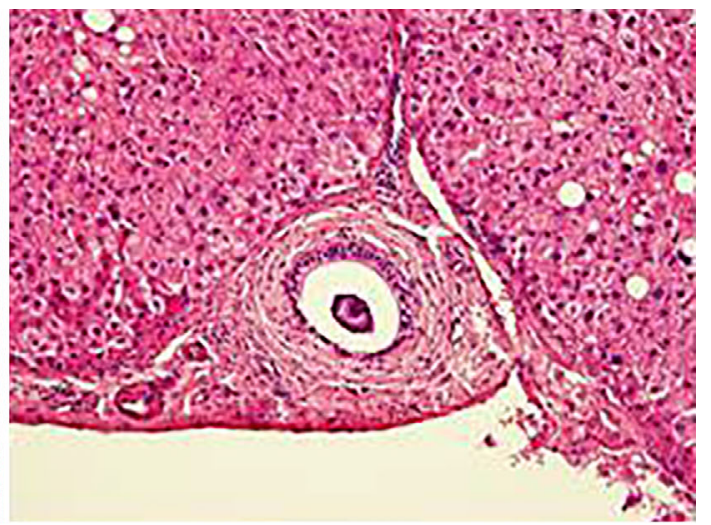

b

Figure 1. Pathological findings of the liver biopsy specimens. (a) On the initial liver biopsy performed in 1997, although an adequate sample from the portal region was available, only eosinophilic infiltration in the sinusoid and lobular necrotic portions was observed [Hematoxylin and Eosin (H\&E) staining, $\times 400$ ]. (b) The second liver biopsy, performed in 1998, revealed onion skin-like fibrosis around the intrahepatic bile ducts $($ H\&E staining, $\times 200)$.

an allergen test was positive for cedar pollen only, ruling the possibility of parasitic disease. In addition, a bone marrow examination showed an increased eosinophil count, although no atypical cells were detected. Prednisolone (PSL) therapy (30 mg/day) was subsequently initiated for the patient's eosinophilia and suspected AIH.

The therapy resulted in improvements in the $\mathrm{IgG}$ level, transaminase level and eosinophil count; however, the biliary enzyme levels remained slightly elevated. The PSL therapy was continued at a reduced dose, and, six months later, a follow-up liver biopsy revealed onion skin-like fibrosis around the intrahepatic bile ducts (Fig. 1b), leading to a diagnosis of PSC. In 2001 (at 30 years of age), the patient presented with right hypochondrial pain and fever and was admitted for a closer examination. Under suspicion of cholangitis, endoscopic retrograde cholangiopancreatography (ERCP) was performed, the results of which revealed irregular narrowing of the intra- and extra-hepatic bile ducts, thus supporting the diagnosis of PSC (Fig. 2a). The administration of ursodeoxycholic acid (UDCA) at a dose of $150 \mathrm{mg}$ / day, in addition to PSL at an increased dose of $25 \mathrm{mg} /$ day, was then started. The dose of PSL was gradually tapered, although abnormal biliary enzyme levels continued to be observed (ALP: $425 \mathrm{IU} / \mathrm{L}$, and GGT: $338 \mathrm{IU} / \mathrm{L}$ ). After two years, the patient developed diarrhea and abdominal pain and consequently underwent colonoscopy at a nearby clinic, which demonstrated edema and erosion of the intestinal mucosa. Furthermore, a colon biopsy performed prior to treatment had shown severe infiltration of eosinophils with no evidence of UC, such as cryptitis, crypt abscesses or basal plasmacytosis, leading to a diagnosis of UC at the clinic. Additional treatment with 5-aminosalicylic acid (5-ASA) was then administered. Although the patient's symptoms gradually improved, he was referred to our hospital in May 2008, and has since been followed up on an outpatient basis at our hospital. Following discontinuation of the therapy with $5 \mathrm{mg} /$ day of PSL and continued treatment with 5-ASA, the patient's symptoms were well controlled until a relapse of diarrhea with increased biliary enzyme levels was observed in July 2008. Magnetic resonance cholangiopancreatography (MRCP) showed no evidence of progressive dilatation or narrowing of the intra- and extra-hepatic bile ducts, with slight irregularity in the diameter of the intrahepatic bile ducts (Fig. 2b). Treatment with PSL at a dose of $5 \mathrm{mg}$ / day was then resumed, and the patient's symptoms stabilized. In February 2010, he again developed abdominal pain and diarrhea (5-6 times/day). Despite increasing the dose of PSL to $20 \mathrm{mg} /$ day, these symptoms did not improve, and the patient was admitted for further treatment.

The physical findings on admission included a body height of $182 \mathrm{~cm}$, body weight of $60.3 \mathrm{~kg}$, body temperature of $36.5^{\circ} \mathrm{C}$, blood pressure of $120 / 60 \mathrm{mmHg}$ and pulse rate of 72 beats/min. The patient's consciousness was clear, with no jaundice in the bulbar conjunctiva, anemia in the palpebral conjunctiva, rales or heart murmurs. The abdomen was flat and soft, with no tenderness. Neither the liver nor spleen were palpable.

The examination findings on admission are summarized in the Table. The WBC count was slightly increased with eosinophilia (12\%). Biochemistry disclosed decreased albumin and increased biliary enzyme levels. A positive ANA test (1:320) and increased IgG level were also noted. On a stool culture test, only indigenous bacteria were detected, with no anti-Entamoeba histolytica antibodies.

Abdominal ultrasonography and CT revealed thickening of the common bile duct wall and mild dilatation of the intrahepatic bile ducts; however, no hepatomegaly/ splenomegaly or intrahepatic tumorous lesions were observed. MRCP showed a mild irregularity in the diameter of the intrahepatic bile ducts, consistent with the findings obtained in July 2008. Colonoscopy demonstrated erosive lesions throughout the large intestine histologically character- 


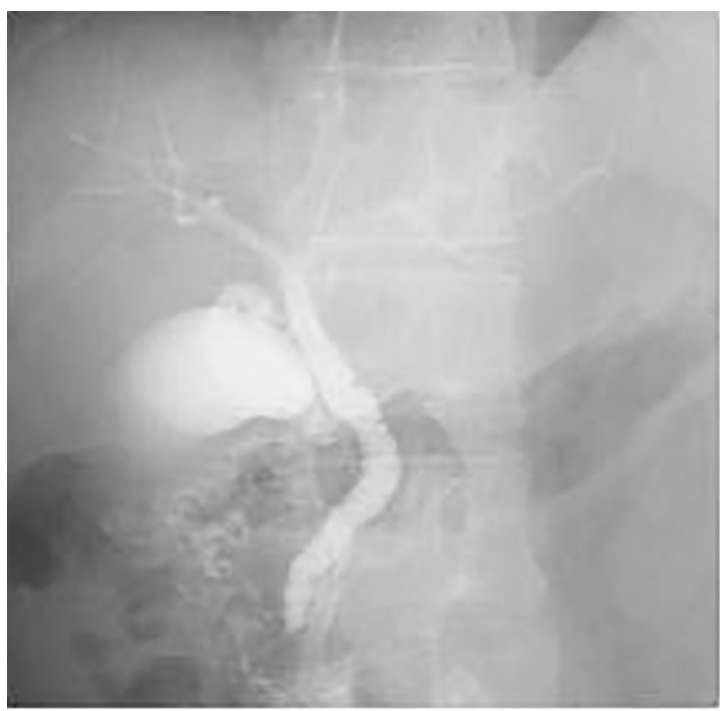

a
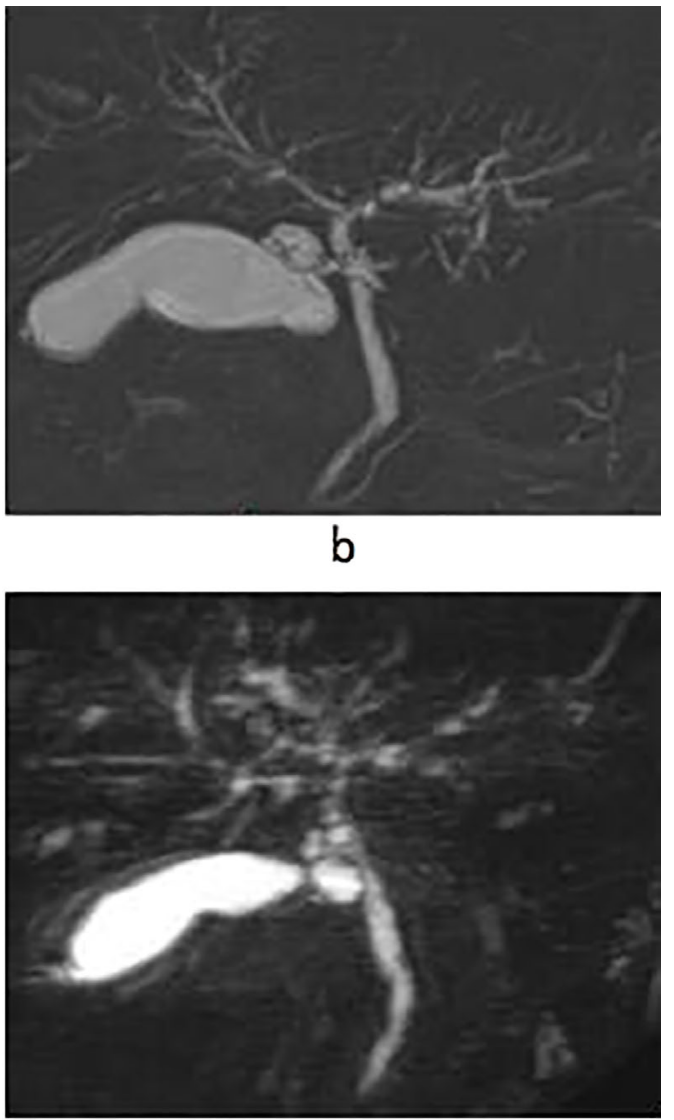

C

Figure 2. ERCP and MRCP images. Irregular narrowing of the intra- and extra-hepatic bile ducts progressively worsened. (a) ERCP performed in 2001. (b) MRCP performed in 2008. (c) MRCP performed in 2012.

Table. Laboratory Data on Admission

\begin{tabular}{lrllrllrl}
\hline WBC & 9,300 & $/ \mu \mathrm{L}$ & TP & 6.9 & $\mathrm{~g} / \mathrm{dL}$ & IgG & 2,107 & $\mathrm{mg} / \mathrm{dL}$ \\
Neut & 45 & $\%$ & Alb & 3.5 & $\mathrm{~g} / \mathrm{dL}$ & $\mathrm{IgA}$ & 285 & $\mathrm{mg} / \mathrm{dL}$ \\
Lymph & 32 & $\%$ & AST & 25 & $\mathrm{IU} / \mathrm{L}$ & $\mathrm{IgM}$ & 108 & $\mathrm{mg} / \mathrm{dL}$ \\
Eosino & 12 & $\%$ & ALT & 17 & $\mathrm{IU} / \mathrm{L}$ & & & \\
RBC & $3.94 \times 10^{4}$ & $/ \mu \mathrm{L}$ & $\mathrm{LDH}$ & 175 & $\mathrm{IU} / \mathrm{L}$ & ANA & $\times 320$ (homogenous) \\
$\mathrm{Hb}$ & 12.1 & $\mathrm{~g} / \mathrm{dL}$ & ALP & 387 & $\mathrm{IU} / \mathrm{L}$ & MPO-ANCA & $(-)$ \\
Hct & 36.2 & $\%$ & $\gamma-\mathrm{GTP}$ & 231 & $\mathrm{IU} / \mathrm{L}$ & PR3-ANCA & $(-)$ \\
Plt & $30.1 \times 10^{4}$ & $/ \mu \mathrm{L}$ & T-Bil & 0.5 & $\mathrm{mg} / \mathrm{dL}$ & & & \\
& & & D-Bil & 0.1 & $\mathrm{mg} / \mathrm{dL}$ & CMV antigenemia & $(-)$ \\
PT & 66.7 & $\%$ & BUN & 19 & $\mathrm{mg} / \mathrm{dL}$ & Entamoeba histolytica Ab & $(-)$ \\
APTT & 25.1 & $\mathrm{sec}$ & Cre & 0.85 & $\mathrm{mg} / \mathrm{dL}$ & & & \\
& & & CRP & 1.43 & $\mathrm{mg} / \mathrm{dL}$ & CD toxin & & $(-)$ \\
\hline
\end{tabular}

ized by severe eosinophilic infiltration (Fig. 3). Although we investigated the patient's upper gastrointestinal tract using endoscopy, we found no suspicious lesions of eosinophilic enteritis. Biopsies of the stomach and duodenum biopsy were not performed.

The patient was initially suspected to have an impaired liver function due to $\mathrm{AIH}$; however, he was later diagnosed with PSC based on the findings of narrowing and dilatation of bile ducts and liver histology. Moreover, the clinical symptoms of abdominal pain and diarrhea and the colonoscopic findings of severe eosinophilic infiltration suggested that he had developed eosinophilic colitis concurrently with PSC, resulting in the aggravation of the symptoms of enteritis. The patient was therefore treated with methylprednisolone at a dose of $250 \mathrm{mg} /$ day for three days, followed by oral PSL at a dose of $40 \mathrm{mg}$. Blood test results showed reduced inflammation, and the patient's diarrhea improved. The abdominal symptoms remained well controlled, even after tapering the dose of PSL. The patient was then followed up on an outpatient basis. However, the abnormal biliary enzyme levels persisted, and in September 2012 MRCP revealed progressive dilatation and narrowing of the intrahe- 


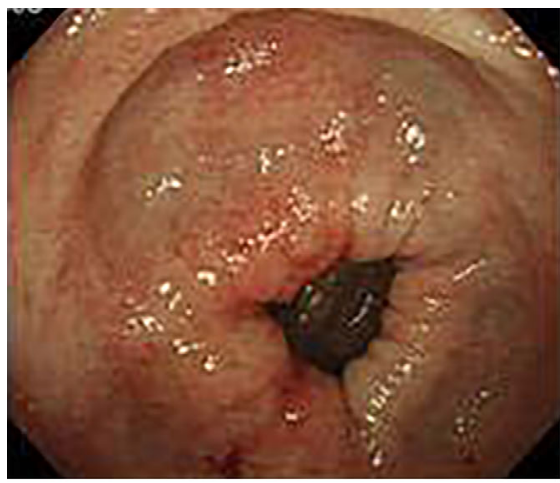

a

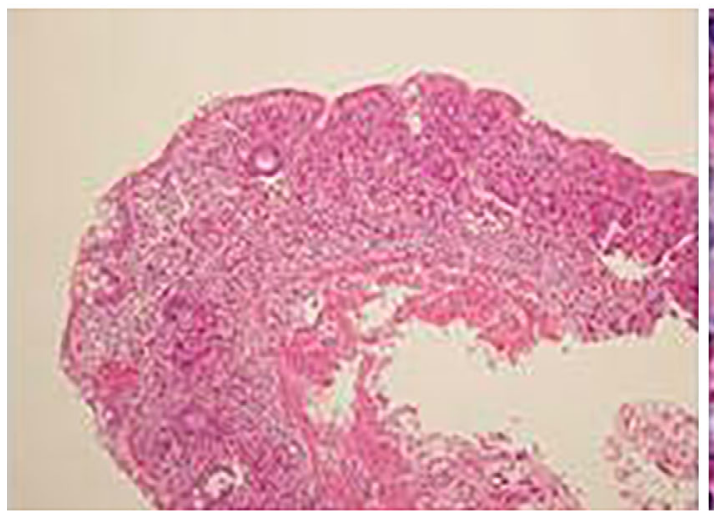

C

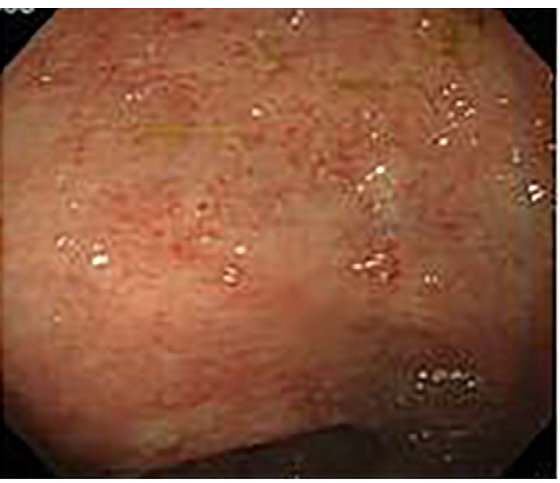

b

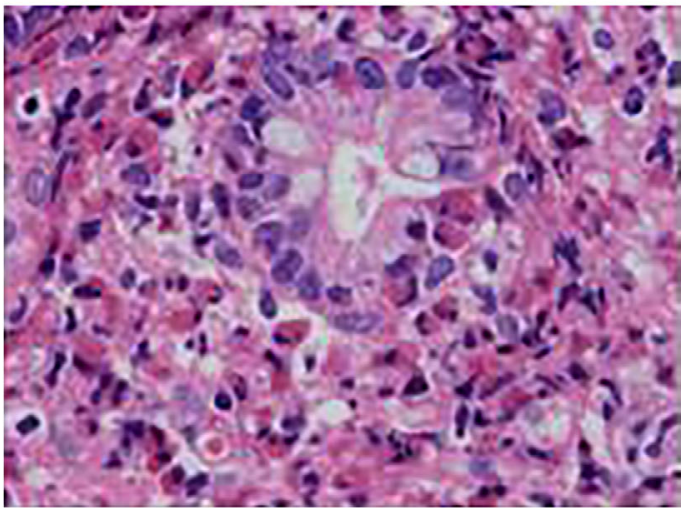

d

Figure 3. Colonoscopic images and histological findings of a biopsy specimen of the large intestine. Colonoscopy revealed erosive lesions throughout the large intestine, and the histological examination of the colon biopsy specimen revealed severe eosinophilic infiltration. No findings suggestive of ulcerative colitis, such as crypt abscesses, crypt distortion or basal plasmacytosis, were observed. (a) Transverse colon. (b) Ascending colon. (c) Biopsy of the transverse colon [Hematoxylin and Eosin (H\&E) staining, $\times 40]$. (d) Biopsy of the ascending colon $(H \& E$ staining, $\times 400)$.

patic bile ducts (Fig. 2c). The above clinical course is summarized in Fig. 4.

\section{Discussion}

In the present case, a diagnosis of AIH was initially suspected based on the examination findings obtained in 1997, including a positive ANA titer, abnormal liver function parameters and an increased IgG level. Nevertheless, the initial liver histology was characterized primarily by eosinophilic infiltration, and no typical findings of $\mathrm{AIH}$, such as interface hepatitis, plasma cell infiltration or rosette formation, were observed. Although a possible effect of PSL therapy was also considered, onion skin-like periductal fibrosis was observed around the bile ducts on the second liver biopsy, which, combined with the MRCP findings, supported the diagnosis of PSC. It has been previously reported that approximately $36 \%$ of Japanese patients with PSC are positive for ANA, some of whom also have increased IgG levels (2).

Regarding eosinophilic colitis, the present patient initially exhibited findings of non-specific chronic inflammation that responded to steroid therapy. In addition, the histological examination performed at symptom relapse revealed eosino- philic infiltration, which satisfied the diagnostic criteria for eosinophilic colitis (6). Furthermore, although prednisolone (PSL) therapy was started for eosinophilia and suspected $\mathrm{AIH}$, the patient developed eosinophilic colitis, resulting in the aggravation of the symptoms of enteritis. According to this clinical course, we judged this case as involving steroiddependent eosinophilic colitis. In fact, steroid dependence has been reported in some patients with eosinophilic colitis (7). In contrast, concurrent PSC and eosinophilic colitis, as noted in the present report, has only been reported in two previous cases $(8,9)$. However, considering that PSC is often associated with eosinophilia (10-12) and that some cases may be reported as unclassifiable enteritis or ulcerative colitis with an increased eosinophil count $(2,13)$, additional similar cases may have been overlooked and consequently not reported. The mechanism underlying the development of concurrent PSC and eosinophilic enteritis has not been elucidated to date. However, possible mechanisms include the involvement of factors that promote the proliferation of eosinophils in the bone marrow and their migration to inflammatory tissue, as well as the expression of eosinophilic granular proteins at affected sites, including eosinophil peroxidase (EPO) $(14,15)$. These factors were not assessed in 


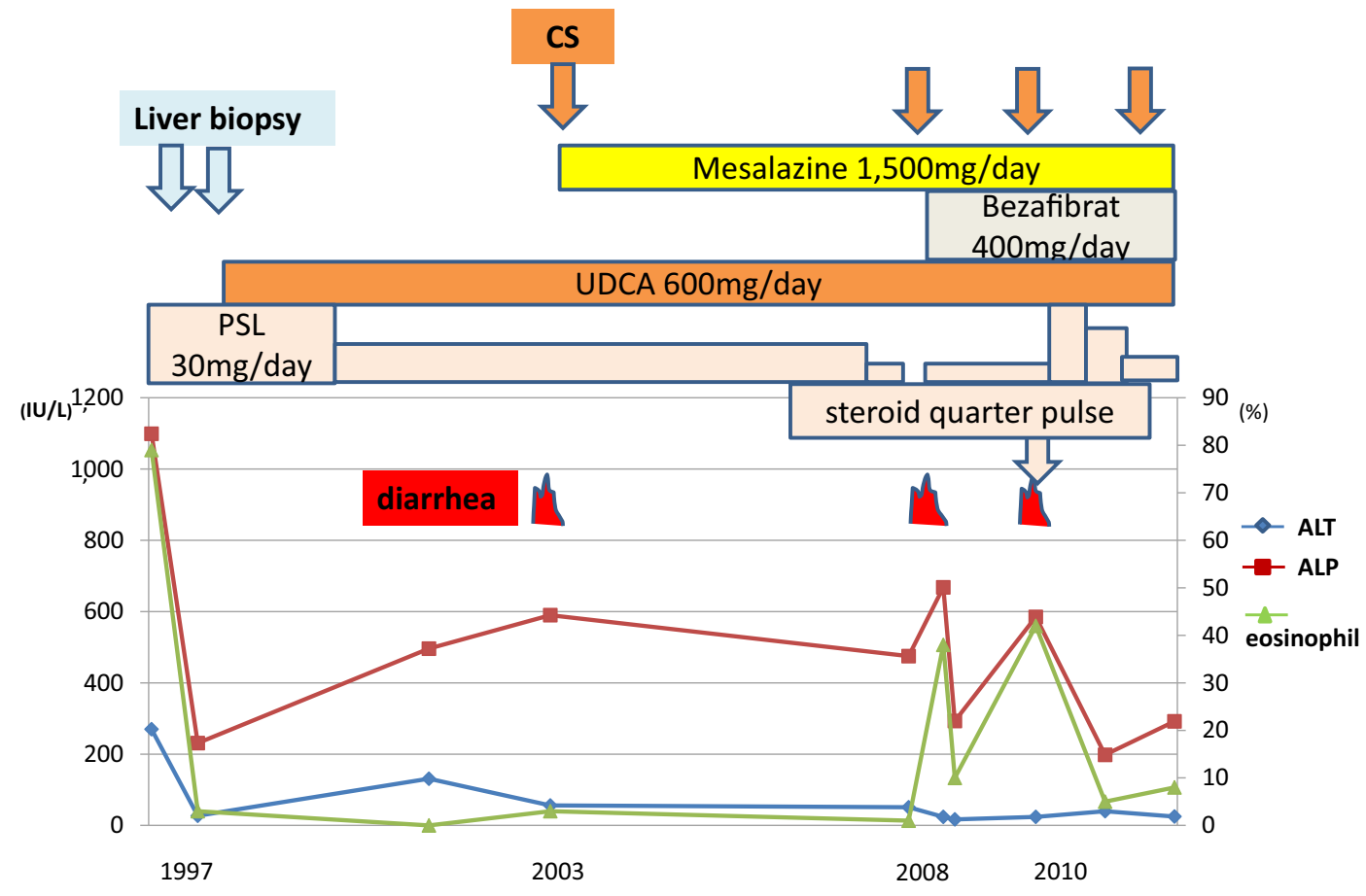

Figure 4. Clinical course of the patient. CS: colonoscopy, UDCA: ursodeoxycholic acid, PSL: prednisolone, ALT: alanine aminotransferase, ALP: alkaline phosphatase

relationship to eosinophilia in the present case.

In the current case, eosinophilic cholangitis is another differential diagnosis of PSC. Eosinophilic cholangitis is a relatively rare disease histologically characterized by inflammatory cell infiltration into the bile ducts, primarily by eosinophils. This condition is not easily distinguished from PSC, which is also associated with an increased eosinophil count and similar imaging findings (16). A diagnosis of eosinophilic cholangitis cannot be ruled out in this case because a bile duct biopsy was not performed; however, this possibility is unlikely based on the liver biopsy findings and the fact that the abnormal biliary enzyme levels persisted, even after treatment with PSL. Miura et al. reported a case of eosinophilic cholangitis associated with periductal fibrosis on histology (17). Since periductal fibrosis does not present with an onion skin-like pattern, a typical finding of PSC, the case reported by Miura et al. is not comparable to the present case. However, in some cases involving patients with eosinophilic cholangitis without peripheral eosinophilia, making the differential diagnosis of PSC is difficult without performing a bile duct biopsy. Eosinophilic cholangiopathy may also be confused with PSC with eosinophilia. IgG4related sclerosing cholangitis is another potential differential diagnosis, although it was unlikely in this case, as the serum IgG4 level remained within the normal range during the course of treatment and no positive results were obtained on the liver biopsy with IgG4 immunostaining. Concurrent eosinophilic cholangitis and eosinophilic gastroenteritis has been reported in six other cases (18-23). Although the imaging findings of the bile duct lesions in these cases are similar to those of PSC, all of the patients responded well to treatment with corticosteroids, which is not consistent with the present case.

We herein presented a rare case of concurrent PSC and eosinophilic colitis. Further accumulation of similar cases is warranted.

The authors state that they have no Conflict of Interest (COI).

\section{Acknowledgement}

We thank Dr. Masamitsu Unagami (Watari Hospital, Japan) and associate professor Kazuhiro Tasaki (Department of Pathology, Fukushima Medical University, Japan) for their valuable opinions and concurrent diagnosis.

\section{References}

1. Takikawa H, Manabe T. Primary sclerosing cholangitis in Japananalysis of 192 cases. J Gastroenterol 32: 134-137, 1997.

2. Takikawa H, Takamori Y, Tanaka A, Kurihara H, Nakanuma Y. Analysis of 388 cases of primary sclerosing cholangitis in Japan; Presence of a subgroup without pancreatic involvement in older patients. Hepatol Res 29: 153-159, 2004.

3. Yan BM, Shaffer EA. Primary eosinophilic disorders of the gastrointestinal tract. Gut 58: 721-732, 2009.

4. Rothenberg ME. Eosinophilic gastrointestinal disorders (EGID). J Allergy Clin Immunol 113: 11-28, 2004.

5. Kinoshita Y, Furuta K, Ishimaura N, et al. Clinical characteristics of Japanese patients with eosinophilic esophagitis and eosinophilic gastroenteritis. J Gastroenterol 48: 333-339, 2013.

6. Talley NJ, Shorter RG, Phillips SF, Zinsmeister AR. Eosinophilic gastroenteritis: a clinicopathological study of patients with disease of the mucosa, muscle layer, and subserosal tissues. Gut 31: 5458, 1990.

7. Ohira G, Matsui Y, Urashima T, Usui A, Taniguchi T, Ochiai T. A case of eosinophilic enteritis requiring emergency surgery. Nihon Shokaki Geka Gakkai Zasshi (J Gastroenterol Surg) 39: 15341539, 2006 (in Japanese, Abstract in English). 
8. Uchida N, Ezaki T, Fukuma H, et al. Concomitant colitis associated with primary sclerosing cholangitis. J Gastroenterol 38: 482487, 2003.

9. Hirano H, Koizumi M, Meguro T, et al. Three cases of primary sclerosing cholangitis. Tando (J Jpn Biliary Asso) 4: 476-483, 1990 (in Japanese, Abstract in English).

10. Neeman A, Kadish U. Marked eosinophilia in a patient with primary sclerosing cholangitis. Am J Med 83: 378-379, 1987.

11. Watanabe H, Ohira H, Kuroda M, et al. Primary sclerosing cholangitis with marked eosinophilic infiltration in the liver. J Gastroenterol 30: 524-528, 1995.

12. Ichikawa $N$, Taniguchi A, Akama H, et al. Sclerosing cholangitis associated with hypereosinophilic syndrome. Intern Med 36: 561564, 1997.

13. Barrie A, Mourabet ME, Weyant $\mathrm{K}$, et al. Recurrent blood eosinophilia in ulcerative colitis is associated with severe disease and primary sclerosing cholangitis. Dig Dis Sci 58: 222-228, 2013.

14. Hogan SP, Rothenberg ME. Review article: The eosinophil as a therapeutictarget in gastrointestinal disease. Aliment Pharmacol Ther 20: 1231-1240, 2004

15. Forbes E, Murase T, Yang M, et al. Immunopathogenesis of experimental ulcerative colitis is mediated by eosinophilperoxidase. J Immunol 172: 5664-5675, 2004.

16. Horiuchi K, Kakizaki S, Kosone T, et al. Marked eosinophilia as the first manifestation of sclerosing cholangitis. Intern Med 48: 1377-1382, 2009.

17. Miura F, Asano $\mathrm{T}$, Amano $\mathrm{H}$, et al. Resected case of eosinophilic cholangiopathy presenting with secondary sclerosing cholangitis. World J Gastroenterol 15: 1394-1397, 2009.

18. Jeyamani R, Ramakrishna BS, Kurian G. Eosinophilic cholangiopathy -a report of two cases. Indian J Gastroenterol 26: 190191, 2007.

19. Duseja A, Nada R, Dhiman RK, et al. Eosinophilic cholangiopathy-a case report. Dig Dis Sci 50: 1422-1425, 2005.

20. Jimenez-Saenz M, Villar-Rodriguez JL, Torres Y, et al. Biliary tract disease: a rare manifestation of eosinophilic gastroenteritis. Dig Dis Sci 48: 624-627, 2003.

21. Kuniyoshi M, Ikeda $\mathrm{K}$, Takano $\mathrm{S}$, et al. Case of eosinophilic gastroenteritis complicated with eosinophilic cholecysto-cholangitis. Nihon Naika Gakkai Zasshi (Journal of Japanese Society of Internal Medicine) 87: 1563-1565, 1998 (in Japanese).

22. Schoonbroodt D, Horsmans Y, Laka A, Geubel AP, Hoang P. Eosinophilic gastroenteritis presenting with colitis and cholangitis. Dig Dis Sci 40: 308-314, 1995.

23. Scheurlen M, Mörk H, Weber P. Hypereosinophilic syndrome resembling chronic inflammatory bowel disease with primary sclerosing cholangitis. J Clin Gastroenterol 14: 59-63, 1992.

(C) 2014 The Japanese Society of Internal Medicine http://www.naika.or.jp/imonline/index.html 